\title{
Reduced-Complexity Approx-Log-MAP and Max-Log-MAP Soft PSK/QAM Detection Algorithms
}

\author{
Chao Xu, Student Member, IEEE, Dandan Liang, Student Member, IEEE, Shinya Sugiura, Senior Member, IEEE, \\ Soon Xin $\mathrm{Ng}$, Senior Member, IEEE, and Lajos Hanzo* Fellow, IEEE
}

\begin{abstract}
In this paper, we propose to reduce the complexity of both the Approx-Log-MAP algorithm as well as of the Max-LogMAP algorithm conceived for generalized PSK/QAM detection, where only a reduced-size subset of the PSK/QAM constellation points is taken into account for producing a single soft-bit output. Although the detectors of Gray-labelled low-order PSK/QAM schemes generally produce near-horizontal EXIT curves, our proposed detectors exploit the a priori LLRs gleaned from a channel decoder in order to retain the optimum detection capability for all PSK/QAM constellations. Furthermore, we demonstrate in this paper that the widely applied MIMO schemes including VBLAST and STBC, which invoke the proposed soft PSK/QAM detectors may also benefit from our reduced-complexity design. Our simulation results confirm that a near-capacity performance may be achieved by the proposed detectors at a substantially reduced detection complexity.
\end{abstract}

Index Terms-Reduced-complexity, Approx-Log-MAP, MaxLog-MAP, Turbo detection, Bit metric generation, Gray-labelling, PSK, Square QAM, Star QAM, Cross QAM, V-BLAST, STBC.

\section{INTRODUCTION}

$\mathbf{T}$ HE significant technical breakthrough of Turbo Codes (TCs) was proposed in [1], where a substantial performance improvement was achieved by exchanging extrinsic information between two channel code decoders. Inspired by the development of TC, the PSK/QAM demodulator was modified in [2], [3] so that turbo detection may be carried out by exchanging extrinsic information between the outer channel decoder and the inner PSK/QAM demodulator. In order to eliminate the error floor often observed in twocomponent concatenated codes, it was proposed in [4] that a further Unity Rate Code (URC) may be incorporated, so that an infinitesimally low BER may be achieved by a threestage turbo detector. Furthermore, the IRregular Convolutional Code (IRCC) concept [5], [6] was proposed for replacing the regular convolutional codes, so that a vanishingly low BER may be achieved at a near-capacity SNR.

As researchers inch closer and closer to the channel capacity, the complexity of the resultant communication systems is also increased. In fact, soft-decision-aided MIMO detection typically contributes a substantial fraction of the total complexity. In order to circumvent this problem, on one hand,

The authors are with the School of Electronics and Computer Science, University of Southampton, Southampton, SO17 1BJ, U.K. (e-mail: $\{$ cx1g08,dl4e08,sxn,lh\}@ecs.soton.ac.uk).

Shinya Sugiura is currently with the Toyota Central R\&D Labs, Inc., Aichi 480-1192, Japan (e-mail:sugiura@ieee.org).

The financial support of the RC-UK under the auspices of the India-UK Advanced Technology Centre (IU-ATC) and that of the EPSRC under the China-UK science bridge as well as that of the EU's Concerto project is gratefully acknowledged. often low-complexity linear receivers (e.g. MMSE receiver) [7]-[9] are employed for multiple-stream MIMO schemes (e.g. V-BLAST [10]) in order to separate the superimposed parallel data streams. On the other hand, the parallel streams of orthogonal STBC schemes [11], [12] may be readily transformed into an equivalent single-stream form, as a benefit of the orthogonal space-time code design. Therefore, when the conventional $L$ PSK/QAM detector is invoked by the MIMO receivers, the detection complexity is on the order of $O(L)$ instead of $O\left(L^{Q}\right)$, where $Q$ represents the number of symbols transmitted together. Moreover, the bit-metric generation methods introduced in [13]-[15] may further reduce the complexity order to $O\left(\log _{2} L\right)$, where the approxmiated LLR values are efficiently evaluated on a bit-by-bit basis. However, these early contributions on bit-metric generation did not consider the a priori LLRs. This is because the detection of the Graylabelled low-order PSK/QAM schemes (e.g BPSK/QPSK and Square 16QAM) generally produces near-horizontal curves in the EXIT chart [5], which means that exchanging information between the soft PSK/QAM detector and the channel decoder may have a negligible benefit.

At the time of writing, high-order LPSK/QAM schemes are routinely utilized in commercialized systems. For example, Square 64QAM and Square 256QAM have been included in the ITU-R IMT Advanced 4G standards [16] and in IEEE 802.11ac [17], respectively. As the number of modulation levels $L$ increases, the soft $L P S K / Q A M$ detectors become capable of producing an improved iteration gain. Therefore, a significant performance improvement is attained, once the a priori LLRs have been taken into account by the soft PSK/QAM detector. However, how to relate each a priori LLR to a reduced-size fraction of the channel's output signal constellations remains an open problem. Against this background, the novel contributions of this paper are as follows:

1) We observe that the Max-Log-MAP algorithm aims for finding the maximum probabilities, which is similar to the action of hard PSK/QAM detection. Therefore, after linking each a priori LLR to a reduced-size fraction of the channel's output signal constellations, the MaxLog-MAP algorithm may be operated at a reduced complexity.

2) Furthermore, the corresponding reduced-complexity Approx-Log-MAP algorithm is also conceived by compensating for the Max-Log-MAP algorithm's widelyused Jacobian approximation relying on a lookup table.

3) We have generalized our detection algorithms for different constellations, including LPSK, Square LQAM, 
Star LQAM and Cross LQAM. The symmetry exhibited by each Gray-labelled constellation diagram is the key to the detection complexity reduction.

4) Finally, we demonstrate the benefits of our solution in the context of both channel-coded V-BLAST systems as well as channel-coded STBC systems. The iteration gain achieved by the soft QAM detectors results in a significant performance improvement, while a substantial complexity reduction is achieved without any performance loss.

The remainder of this paper is organized as follows. The VBLAST system and the STBC system are reviewed in Sec. II. In Sec. III, our reduced-complexity soft PSK/QAM detection algorithms are proposed. Our performance results are provided in Sec. IV, while the application of our reduced-complexity design is discussed in Sec. V. Our conclusions are offered in Sec. VI.

\section{MIMO SYSTEMS}

\section{A. V-BLAST Systems}

The architecture of a classic V-BLAST system may be found in [7]. The encoded source data stream is split into multiple streams in order to be modulated and transmitted independently by $M$ transmit antennas. The signal received at the $N$ receive antennas may be expressed as:

$$
\mathbf{Y}_{n}=\mathbf{S}_{n} \mathbf{H}_{n}+\mathbf{V}_{n}=S_{n}^{m} \mathbf{H}_{n}^{m}+\mathbf{S}_{n}^{\bar{m}} \mathbf{H}_{n}^{\bar{m}}+\mathbf{V}_{n}
$$

where $\mathbf{Y}_{n} \in \mathbb{C}^{1 \times N}, \mathbf{S}_{n} \in \mathbb{C}^{1 \times M}, \mathbf{H}_{n} \in \mathbb{C}^{M \times N}$ and $\mathbf{V}_{n} \in \mathbb{C}^{1 \times N}$ refer to the received signal vector, the transmitted LPSK/QAM symbols vector, the Rayleigh fading channels matrix and the Additive White Gaussian Noise (AWGN) vector, which has a zero mean and a variance of $N_{0}$ in each dimension, respectively. Furthermore, $S_{n}^{m}$ and $\mathbf{H}_{n}^{m} \in \mathbb{C}^{1 \times N}$ denote the $m$-th element in $\mathbf{S}_{n}$ and the $m$-th row in $\mathbf{H}_{n}$, respectively, while $\mathbf{S}_{n}^{\bar{m}} \in \mathbb{C}^{1 \times(M-1)}$ and $\mathbf{H}_{n}^{\bar{m}} \in \mathbb{C}^{(M-1) \times N}$ are obtained by removing the $m$-th element in $\mathbf{S}_{n}$ and by removing the $m$-th row in $\mathbf{H}_{n}$, respectively. It can be seen in Eq. (1) that when $S_{n}^{m}$ is detected, the rest of the transmitted symbols introduce Inter-Antenna Interference (IAI).

The full-search-based ML detection imposes an excessive detection complexity. More explicitly, upon obtaining the a priori LLRs $\left\{L_{a}\left(b_{j}\right)\right\}_{j=1}^{(M \cdot \operatorname{BPS})}$ from the channel decoder, the $a$ posteriori LLRs produced by the Log-MAP algorithm conceived for ML V-BLAST detection may be expressed as [18]:

$$
L_{p}\left(b_{k} \mid \mathbf{Y}_{n}\right)=\ln \left[\frac{\sum_{\mathbf{S}^{i} \in \mathbf{S}_{b_{k}=1}} \exp \left(d_{i}\right)}{\sum_{\mathbf{S}^{i} \in \mathbf{S}_{b_{k}=0}} \exp \left(d_{i}\right)}\right]=L_{a}\left(b_{k}\right)+L_{e}\left(b_{k}\right),
$$

where $\mathbf{S}_{b_{k}=1}$ and $\mathbf{S}_{b_{k}=0}$ refer to the LPSK/QAM symbol vector sets, when the specific bit $b_{k}$ is fixed to 1 and 0 , respectively. Given a specific $L P S K / Q A M$ symbol vector $\left\{\mathbf{S}^{i}\right\}_{i=1}^{\left(L^{M}\right)}$, its probability metric $\left\{d_{i}\right\}_{i=1}^{\left(L^{M}\right)}$ in Eq. (2) is defined as [2]:

$$
d_{i}=-\frac{\left\|\mathbf{Y}_{n}-\mathbf{S}^{i} \mathbf{H}_{n}\right\|^{2}}{N_{0}}+\sum_{j=1}^{M \cdot \mathrm{BPS}} \widetilde{b}_{j} L_{a}\left(b_{j}\right)
$$

where $\left\{\widetilde{b}_{j}\right\}_{j=1}^{(M \cdot \mathrm{BPS})}$ represents the bit mapping corresponding to the specific $\mathbf{S}^{i}$. It can be seen that Eq. (3) operates on a vector-by-vector basis, which has a complexity order of $O\left(L^{M}\right)$. Therefore, linear matched-filtering detectors [7] may be invoked for detecting the $M$ data streams separately. For example, the low-complexity Zero-Forcing (ZF) filter produces the following output:

$$
\widetilde{\mathbf{Z}}_{n}=\mathbf{Y}_{n} \mathbf{G}_{\mathrm{ZF}}=\mathbf{S}_{n}+\widetilde{\mathbf{V}}_{n},
$$

where the $\mathrm{ZF}$ filter weight matrix is given by $\mathbf{G}_{\mathrm{ZF}}=$ $\mathbf{H}_{n}^{H}\left(\mathbf{H}_{n} \mathbf{H}_{n}^{H}\right)^{-1}$, while the equivalent noise matrix is given by $\mathbf{V}_{n}=\mathbf{V}_{n} \mathbf{G}_{\mathrm{ZF}}$. It can be seen in Eq. (4) that ZF receivers suffer from noise amplification, hence often the Minimum Mean Square Error (MMSE) detector [8], [9] is employed for linear MIMO detection. More explicitly, the MMSE filter designed for detecting the $m$-th transmitted symbol produces the following output:

$$
\begin{aligned}
Z_{n}^{m}=\mathbf{Y}_{n} \mathbf{G}_{\mathrm{MMSE}}^{m}= & S_{n}^{m} \mathbf{H}_{n}^{m} \mathbf{G}_{\mathrm{MMSE}}^{m} \\
& +\mathbf{S}_{n}^{\bar{m}} \mathbf{H}_{n}^{\bar{m}} \mathbf{G}_{\mathrm{MMSE}}^{m}+\mathbf{V}_{n} \mathbf{G}_{\mathrm{MMSE}}^{m},
\end{aligned}
$$

where $\mathbf{G}_{\text {MMSE }}^{m} \in \mathbb{C}^{N \times 1}$ refers to the $m$-th MMSE filter weight matrix. The performance of MMSE detection is limited by the IAI [7], and hence the a priori information is utilized for the sake of interference cancellation, which may be expressed as [9]:

$$
\bar{Z}_{n}^{m}=Z_{n}^{m}-\varepsilon\left\{\mathbf{S}_{n}^{\bar{m}}\right\} \mathbf{H}_{n}^{\bar{m}} \mathbf{G}_{\mathrm{MMSE}}^{m},
$$

where the estimate of a specific symbol based on a priori LLRs is given by:

$\varepsilon\left\{S_{n}^{m}\right\}=\sum_{l=1}^{L} s^{l} \operatorname{Pr}\left(S_{n}^{m}=s^{l}\right)=\sum_{l=1}^{L} s^{l} \cdot \frac{\exp \left[\sum_{j=1}^{\mathrm{BPS}} \widetilde{b}_{j} L_{a}\left(b_{j}\right)\right]}{\prod_{j=1}^{\mathrm{BPS}}\left\{1+\exp \left[L_{a}\left(b_{j}\right)\right]\right\}}$.

It was demonstrated in [8], [9] that $\frac{\partial \varepsilon\left\{\left|\bar{Z}_{n}^{m}-S_{n}^{m}\right|^{2}\right\}}{\partial\left[\left(\mathbf{G}_{\mathrm{MMSE}}^{m}\right)^{H}\right]}=$ 0 results in the MMSE weight matrix shown in Eq. (8), where $\left\{E_{s}^{m}\right\}_{m=1}^{M}$ denotes the transmitted signal power of each antenna, which may be different, if the $M$ transmit antennas belong to $M$ independent users in the context of Space-Division Multiplexing (SDM) [19]. When nonconstant modulus LPSK/QAM is employed, the correlation matrix representing the symbol power is given by $\mathbf{R}_{S S}=$ $\operatorname{diag}\left(\left[\varepsilon\left\{\left|S_{n}^{1}\right|^{2}\right\}, \cdots, \varepsilon\left\{\left|S_{n}^{M}\right|^{2}\right\}\right]\right)$, where the estimate of a specific symbol's power is given by replacing the constellation point $s^{l}$ in Eq. (7) by its power $\left|s^{l}\right|^{2}$. Furthermore, $\mathbf{R}_{S S}^{\bar{m}} \in$ $\mathbb{C}^{(M-1) \times(M-1)}$ in Eq. (8) is obtained by removing both the $m$-th column as well as the $m$-th row in $\mathbf{R}_{S S}$. As a result, the conventional $L \mathrm{QAM}$ detector may be invoked as:

$$
L_{p}\left(b_{k} \mid \mathbf{Y}_{n}\right)=\ln \left[\frac{\sum_{s^{l} \in \mathbf{s}_{b_{k}=1}} \exp \left(d_{l}\right)}{\sum_{s^{l} \in \mathbf{s}_{b_{k}=0}} \exp \left(d_{l}\right)}\right],
$$

where $\mathbf{s}_{b_{k}=1}$ and $\mathbf{s}_{b_{k}=0}$ refer to the LPSK/QAM symbols sets, when the specific bit $b_{k}$ is fixed to 1 and 0 , respectively, while the symbol-by-symbol-based probability metric $\left\{d_{l}\right\}_{l=1}^{L}$ 


$$
\mathbf{G}_{\text {MMSE }}^{m}=\left\{\left(\mathbf{H}_{n}^{m}\right)^{H} \mathbf{H}_{n}^{m}+\frac{\left(\mathbf{H}_{n}^{\bar{m}}\right)^{H}}{E_{s}^{m}}\left[\mathbf{R}_{S S}^{\bar{m}}-\operatorname{diag}\left(\varepsilon\left\{\mathbf{S}_{n}^{\bar{m}}\right\}^{H} \varepsilon\left\{\mathbf{S}_{n}^{\bar{m}}\right\}\right)\right] \mathbf{H}_{n}^{\bar{m}}+\frac{N_{0}}{E_{s}^{m}} \mathbf{I}_{N}\right\}^{-1}\left(\mathbf{H}_{n}^{m}\right)^{H} .
$$

becomes [2]:

$$
d_{l}=-\frac{\left|\widetilde{Z}_{n}^{m}-s^{l}\right|^{2}}{\widetilde{N}_{0}}+\sum_{j=1}^{\text {BPS }} \widetilde{b}_{j} L_{a}\left(b_{j}\right) .
$$

For ZF receivers, $\widetilde{Z}_{n}^{m}$ refers to the m-th element of ZF filter output $\widetilde{Z}_{n}$ of Eq. (4), while the equivalent noise power is given by $\widetilde{N}_{0}=\left\|\mathbf{G}_{\mathrm{ZF}}^{m}\right\|^{2} N_{0}$, where $\mathbf{G}_{\mathrm{ZF}}^{m}$ refers to the $\mathrm{m}$-th column of the ZF filter's weight matrix $\mathbf{G}_{\mathrm{ZF}}$. For MMSE receivers, we have $\widetilde{Z}_{n}^{m}=\bar{Z}_{n}^{m} \cdot\left(\bar{h}_{n}^{m}\right)^{*} /\left|\bar{h}_{n}^{m}\right|^{2}$ and $\widetilde{N}_{0}=$ $\left\|\mathbf{G}_{\mathrm{MMSE}}^{m}\right\|_{{ }^{m}}^{2} \cdot N_{0} /\left|\bar{h}_{n}^{m}\right|^{2}$, where the equivalent fading factor is given by $\bar{h}_{n}^{m}=\mathbf{H}_{n}^{m} \mathbf{G}_{\mathrm{MMSE}}^{m}$.

\section{B. STBC Detection}

For a STBC system, the transmitter firstly encodes the $\left(Q \log _{2} L\right)$ bits to an LPSK/QAM symbols vector of $\mathbf{s}_{n}=$ $\left[s_{n}^{1}, \cdots, s_{n}^{Q}\right]^{T}$. During $T$ symbol periods, the symbol-matrix transmitted from the $M$ transmit antennas may be formulated as:

$$
\mathbf{S}_{n}=\frac{1}{\sqrt{M}} G_{M}\left(\mathbf{s}_{n}\right)
$$

where the $(T \times M)$-element matrix $G_{M}\left(\mathbf{s}_{n}\right)$ represents the orthogonal STBC structure. Considering Alamouti's G2 STBC as an example, the STBC matrix $G_{M}\left(\mathbf{s}_{n}\right)$ is given by [11]:

$$
G_{2}\left(\mathbf{s}_{n}\right)=\left[\begin{array}{cc}
s_{n}^{1} & s_{n}^{2} \\
-\left(s_{n}^{2}\right)^{*} & \left(s_{n}^{1}\right)^{*}
\end{array}\right] .
$$

The signal received by the $N$ receive antennas may still be represented by Eq. (1), but the size of the transmitted signal matrix, the received signal matrix and the AWGN matrix becomes $\mathbf{S}_{n} \in \mathbb{C}^{T \times M}, \mathbf{Y}_{n} \in \mathbb{C}^{T \times N}$ and $\mathbf{V}_{n} \in \mathbb{C}^{T \times N}$, respectively. Furthermore, due to the orthogonality offered by the STBC design, the equivalent received symbol may be expressed as [11], [12]:

$$
z_{n}^{q}=s_{n}^{q} \cdot \widetilde{h}_{n}+\widetilde{v}_{n}^{q} .
$$

More explicitly, the decorrelating variables $\left\{z_{n}^{q}\right\}_{q=1}^{Q}$ for Alamouti's G2 STBC are given by [11]:

$$
\begin{aligned}
& z_{n}^{1}=\mathbf{Y}_{n}^{1}\left(\mathbf{H}_{n}^{1}\right)^{H}+\mathbf{H}_{n}^{2}\left(\mathbf{Y}_{n}^{2}\right)^{H}=s_{n}^{1} \cdot \widetilde{h}_{n}+\widetilde{v}_{n}^{1}, \\
& z_{n}^{2}=\mathbf{Y}_{n}^{1}\left(\mathbf{H}_{n}^{2}\right)^{H}-\mathbf{H}_{n}^{1}\left(\mathbf{Y}_{n}^{2}\right)^{H}=s_{n}^{2} \cdot \widetilde{h}_{n}+\widetilde{v}_{n}^{2},
\end{aligned}
$$

where the equivalent fading factor is given by $\left(\widetilde{h}_{n}=\frac{\left\|\mathbf{H}_{n}\right\|^{2}}{\sqrt{2}}\right)$, while the equivalent noise factors $\left\{\widetilde{v}_{n}^{q}\right\}_{q=1}^{Q}$ have a new variance of $\left(\left\|\mathbf{H}_{n}\right\|^{2} \cdot N_{0}\right)$. The $(1 \times N)$-element vectors $\left\{\mathbf{Y}_{n}^{i}\right\}_{i=1}^{T}$ and $\left\{\mathbf{H}_{n}^{i}\right\}_{i=1}^{M}$ denotes the $i$-th row in the matrices $\mathbf{Y}_{n}$ and $\mathbf{H}_{n}$, respectively.

Based on the new equivalent received signal model of Eq. (13), the $L P S K / Q A M$ symbol probability metric $\left\{d_{l}\right\}_{l=1}^{L}$ seen in Eq. (9) may be expressed as:

$$
d_{l}=-\frac{\left|\widetilde{z}_{n}^{q}-s^{l}\right|^{2}}{\widetilde{N}_{0}}+\sum_{j=1}^{\mathrm{BPS}} \widetilde{b}_{j} L_{a}\left(b_{j}\right),
$$

where we have $\left(\widetilde{z}_{n}^{q}=z_{n}^{q} / \widetilde{h}_{n}\right)$ and $\left(\widetilde{N}_{0}=2 N_{0} /\left\|\mathbf{H}_{n}\right\|^{2}\right)$ for the case of Alamouti's G2 STBC.

\section{Reduced-COMPLEXITy Soft PSK/QAM DETECTION}

\section{A. Conventional Soft PSK/QAM Detection}

In practice the Log-MAP algorithm of Eq. (9) imposes an unaffordable detection complexity, owing to its high-precious representation of probabilities. Therefore, when designing a low-complexity soft detector, the so-called Max-Log-MAP algorithm [18] may be invoked as:

$$
L_{p}\left(b_{k} \mid \mathbf{Y}_{n}\right)=\max _{s^{l} \in \mathbf{s}_{b_{k}=1}}\left(d_{l}\right)-\max _{s^{l} \in \mathbf{s}_{b_{k}=0}}\left(d_{l}\right) .
$$

Since only two maximum probability metrics are taken into account in Eq. (16), the Max-Log-MAP algorithm imposes a slight performance degradation. In order to compensate for this performance loss, the Approx-Log-MAP algorithm [20] was introduced as :

$$
L_{p}\left(b_{k} \mid \mathbf{Y}_{n}\right)=\operatorname{jac}_{s^{l} \in \mathbf{s}_{b_{k}=1}}\left(d_{l}\right)-\mathrm{jac}_{s^{l} \in \mathbf{s}_{b_{k}=0}}\left(d_{l}\right),
$$

where jac denotes the Jacobian algorithm, which may be expressed as [21]:

$$
\operatorname{jac}\left(d_{1}, d_{2}\right)=\max \left\{d_{1}, d_{2}\right\}+\Gamma\left\{\left|d_{1}-d_{2}\right|\right\} .
$$

The addional term of $\Gamma\left\{\left|d_{1}-d_{2}\right|\right\}$ in Eq. (18) takes into account the difference between $d_{1}$ and $d_{2}$ according to a lookup table [21]. Based on the ZF/MMSE aided V-BLAST estimation of Eq. (10) as well as on the STBC estimation of Eq. (15), the symbol probability metrics $\left\{d_{l}\right\}_{l=1}^{L}$ invoked by the detection algorithms may be summarized as:

$$
d_{l}=-\frac{\left|\widetilde{z}_{n}-s^{l}\right|^{2}}{\widetilde{N}_{0}}+\sum_{j=1}^{\text {BPS }} \widetilde{b}_{j} L_{a}\left(b_{j}\right) .
$$

When Square LQAM is employed, the real and the imaginary parts of the constellation may be detected separately. As a result, the Square $L$ QAM symbol probability metrics $\left\{d_{l}^{\operatorname{Im}}\right\}_{l=1}^{\sqrt{L}}$ and $\left\{d_{l}^{\operatorname{Re}}\right\}_{l=1}^{\sqrt{L}}$ are given by:

$$
\begin{aligned}
& d_{l}^{\operatorname{Im}}=-\frac{\left|\operatorname{Im}\left(\widetilde{z}_{n}\right)-\operatorname{Im}\left(s^{l}\right)\right|^{2}}{\widetilde{N}_{0}}+\sum_{j=1}^{\mathrm{BPS} / 2} \widetilde{b}_{j} L_{a}\left(b_{j}\right), \\
& d_{l}^{\mathrm{Re}}=-\frac{\left|\operatorname{Re}\left(\widetilde{z}_{n}\right)-\operatorname{Re}\left(s^{l}\right)\right|^{2}}{\widetilde{N}_{0}}+\sum_{j=\mathrm{BPS} / 2+1}^{\mathrm{BPS}} \widetilde{b}_{j} L_{a}\left(b_{j}\right) .
\end{aligned}
$$

\section{B. Reduced-Complexity Soft Square QAM Detection}

For producing a single soft-bit output, the conventional Max-Log-MAP algorithm of Eq. (16) as well as the ApproxLog-MAP algorithm of Eq. (17) have to estimate and compare all the $\sqrt{L}$ probability metrics using Eq. (20) according to the $\sqrt{L}$ PAM constellation, which forms the real and imaginary part of a Square $L \mathrm{QAM}$ symbol. In this section, we aim for reducing the detection complexity by reducing the number of constellation points that have to be visited. 


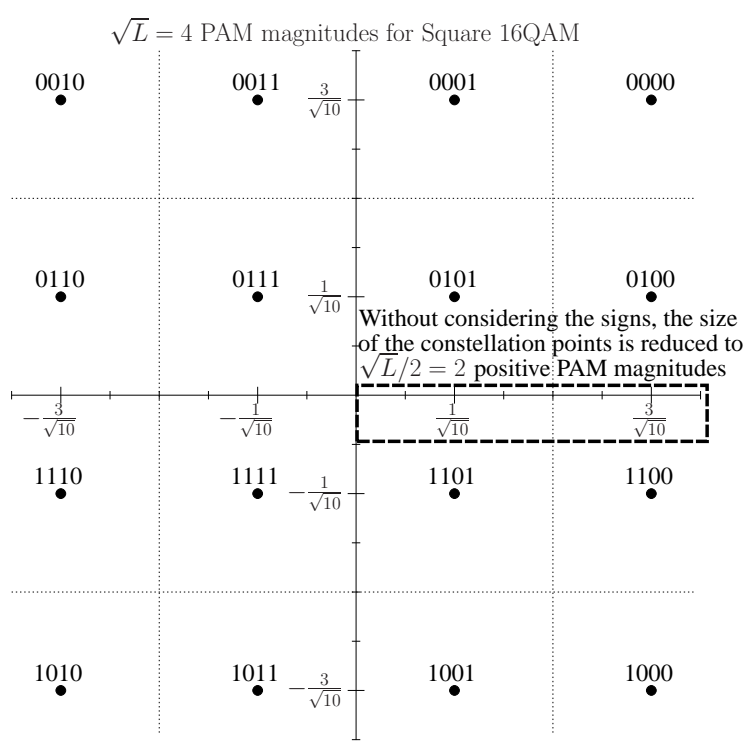

Fig. 1. Constellation diagram of Square 16QAM. The real and the imaginary parts of a Square $L$ QAM symbol may always be detected separately.

First of all, let us extend the imaginary part probability metric calculation of Eq. (20) as:

$d_{l}^{\operatorname{Im}}=-\frac{\left|\operatorname{Im}\left(\widetilde{z}_{n}\right)\right|^{2}}{\widetilde{N}_{0}}-\frac{\left|\operatorname{Im}\left(s^{l}\right)\right|^{2}}{\widetilde{N}_{0}}+\frac{2 \operatorname{Im}\left(\widetilde{z}_{n}\right) \operatorname{Im}\left(s^{l}\right)}{\widetilde{N}_{0}}+\sum_{j=1}^{\mathrm{BPS} / 2} \widetilde{b}_{j} L_{a}\left(b_{j}\right)$,

where $\left(-\frac{\mid \operatorname{Im}\left(\widetilde{z}_{n}\right)^{2}}{\widetilde{N}_{0}}\right)$ is invariant over the candidate variable $s^{l}$, which may be ignored because all the multiplicative constants may be eliminated by the division operation in the Log-MAP algorithm of Eq. (9). Therefore, Eq. (21) may be further simplified as:

$$
d_{l}^{\operatorname{Im}}=\frac{\operatorname{Im}\left(s^{l}\right)}{\bar{N}_{0}} \operatorname{Im}\left(\widetilde{z}_{n}\right)-\frac{\left|\operatorname{Im}\left(s^{l}\right)\right|^{2}}{\widetilde{N}_{0}}+\sum_{j=1}^{\mathrm{BPS} / 2} \widetilde{b}_{j} L_{a}\left(b_{j}\right),
$$

where we have $\left(\bar{N}_{0}=\widetilde{N}_{0} / 2\right)$. Let us now take the Square 16QAM constellation of Fig. 1 as an example, where the $(\sqrt{L}=4)$ imaginary metrics of Eq. (22) may be expressed as:

$$
\begin{aligned}
d_{1}^{\operatorname{Im}} & =\frac{3 \operatorname{Im}\left(\widetilde{z}_{n}\right)}{\sqrt{10} \cdot \bar{N}_{0}}-\frac{9}{10 N_{0}} \\
& =t_{\operatorname{Im} 2}-\frac{4}{5 N_{0}}+C_{\operatorname{Im}}, \\
d_{2}^{\operatorname{Im}} & =\frac{\operatorname{Im}\left(\widetilde{z}_{n}\right)}{\sqrt{10} \cdot \bar{N}_{0}}-\frac{1}{10 N_{0}}+L_{a}\left(b_{2}\right) \\
& =t_{\operatorname{Im} 1}+L_{a}\left(b_{2}\right)+C_{\operatorname{Im}}, \\
d_{3}^{\operatorname{Im}} & =-\frac{3 \operatorname{Im}\left(\widetilde{z}_{n}\right)}{\sqrt{10} \cdot \bar{N}_{0}}-\frac{9}{10 \widetilde{N}_{0}}+L_{a}\left(b_{1}\right) \\
& =-t_{\operatorname{Im} 2}-\frac{4}{5 \widetilde{N}_{0}}+C_{\operatorname{Im}}, \\
d_{4}^{\operatorname{Im}} & =-\frac{\operatorname{Im}\left(\widetilde{z}_{n}\right)}{\sqrt{10} \cdot \overline{N_{0}}}-\frac{1}{10 \tilde{N}_{0}}+L_{a}\left(b_{1}\right)+L_{a}\left(b_{2}\right) \\
& =-t_{\operatorname{Im} 1}+L_{a}\left(b_{2}\right)+C_{\operatorname{Im}},
\end{aligned}
$$

where we relate the imaginary part of $\widetilde{z}_{n}$ to the corresponding soft bit input $L_{a}\left(b_{1}\right)$ as:

$$
t_{\mathrm{Im} 1}=\frac{\operatorname{Im}\left(\widetilde{z}_{n}\right)}{\sqrt{10} \cdot \bar{N}_{0}}-\frac{L_{a}\left(b_{1}\right)}{2}, \quad t_{\operatorname{Im} 2}=\frac{3 \operatorname{Im}\left(\widetilde{z}_{n}\right)}{\sqrt{10} \cdot \bar{N}_{0}}-\frac{L_{a}\left(b_{1}\right)}{2},
$$

while the constant is given by $\left[C_{\operatorname{Im}}=-\frac{1}{10 N_{0}}+\frac{L_{a}\left(b_{1}\right)}{2}\right]$. As a result, the maximum metric found by evaluating all the four candidates in Eq. (23), which is pursued by the Max-Log-MAP of Eq. (16), may be obtained by:

$$
\begin{aligned}
d_{\max }^{\mathrm{Im}} & =\max \left\{\begin{array}{l}
\max \left\{d_{2}^{\mathrm{Im}}, d_{4}^{\mathrm{Im}}\right\} \\
\max \left\{d_{1}^{\mathrm{Im}}, d_{3}^{\operatorname{Im}}\right\}
\end{array}\right\} \\
& =\max \left\{\begin{array}{l}
\left|t_{\mathrm{Im} 1}\right|+L_{a}\left(b_{2}\right) \\
\left|t_{\operatorname{Im} 2}\right|-\frac{4}{5 \tilde{N}_{0}}
\end{array}\right\}+C_{\mathrm{Im}} .
\end{aligned}
$$

Therefore, instead of evaluating and comparing Eq. (22) four times in Eq. (23) according to the 4PAM constellation points, Eq. (25) is obtained by comparing two candidates, where the absolute value calculation eliminates the need for considering the signs, as portrayed by Fig. 1 .

According to the maximum metric search approach demonstrated in Eq. (25), the first two soft bits produced by the reduced-complexity Max-Log-MAP algorithm may be obtained as:

$$
\begin{aligned}
L_{p}\left(b_{1}\right) & =\max \left\{d_{3}^{\mathrm{Im}}, d_{4}^{\mathrm{Im}}\right\}-\max \left\{d_{1}^{\mathrm{Im}}, d_{2}^{\mathrm{Im}}\right\} \\
& =\max \left\{\begin{array}{l}
-t_{\mathrm{Im} 1}+L_{a}\left(b_{2}\right) \\
-t_{\operatorname{Im} 2}-\frac{4}{5 \widetilde{N}_{0}}
\end{array}\right\}-\max \left\{\begin{array}{c}
t_{\mathrm{Im} 1}+L_{a}\left(b_{2}\right) \\
t_{\mathrm{Im} 2}-\frac{4}{5 \tilde{N}_{0}}
\end{array}\right\}, \\
L_{p}\left(b_{2}\right) & =\max \left\{d_{2}^{\mathrm{Im}}, d_{4}^{\mathrm{Im}}\right\}-\max \left\{d_{1}^{\mathrm{Im}}, d_{3}^{\mathrm{Im}}\right\} \\
& =\left|t_{\mathrm{Im} 1}\right|+L_{a}\left(b_{2}\right)-\left|t_{\mathrm{Im} 2}\right|+\frac{4}{5 \widetilde{N}_{0}} .
\end{aligned}
$$

Similarly, the corresponding Approx-Log-MAP algorithm may be obtained by compensating the error imposed by considering only the maximum in Eq. (26) as:

$$
\begin{aligned}
& L_{p}\left(b_{1}\right)=\mathrm{jac}\left\{\begin{array}{l}
-t_{\mathrm{Im} 1}+L_{a}\left(b_{2}\right) \\
-t_{\mathrm{Im} 2}-\frac{4}{5 \widetilde{N}_{0}}
\end{array}\right\}-\mathrm{jac}\left\{\begin{array}{l}
t_{\mathrm{Im} 1}+L_{a}\left(b_{2}\right) \\
t_{\mathrm{Im} 2}-\frac{4}{5 \widetilde{N}_{0}}
\end{array}\right\}, \\
& L_{p}\left(b_{2}\right)=\Lambda\left(\left|t_{\mathrm{Im} 1}\right|\right)+L_{a}\left(b_{2}\right)-\Lambda\left(\left|t_{\mathrm{Im} 2}\right|\right)+\frac{4}{5 \widetilde{N}_{0}}
\end{aligned}
$$

where we define the special case of the Jacobian algorithm of Eq. (18) as:

$$
\Lambda(|t|)=\operatorname{jac}(t,-t)=|t|+\Gamma\{2|t|\} .
$$

Based on the example of Square 16QAM detection, we summarize the Max-Log-MAP algorithm conceived for Square $L \mathrm{QAM}$ detection as follows:

\section{Algorithm 1: Max-Log-MAP Algorithm for Square $L$ QAM Detection.}

1) Define the test-variables, which relate $L_{a}\left(b_{\mathrm{BPS} / 2+1}\right)$ and $L_{a}\left(b_{1}\right)$ to the real and imaginary parts of $\widetilde{z}_{n}$ as:

$$
\begin{aligned}
t_{\mathrm{Re}_{i}} & =\frac{A_{i} \operatorname{Re}\left(\widetilde{z}_{n}\right)}{\bar{N}}-\frac{L_{a}\left(b_{\mathrm{BPS} / 2+1}\right)}{2}, \\
t_{\mathrm{Im}_{i}} & =\frac{\left.A_{i} \operatorname{Im}_{z_{n}}\right)}{\bar{N}_{0}}-\frac{L_{a}\left(b_{1}\right)}{2},
\end{aligned}
$$

where $\left\{A_{i}\right\}_{i=1}^{\sqrt{L} / 2}$ are the positive real PAM magnitudes on the $\mathrm{x}$-axis and $\mathrm{y}$-axis of Square LQAM constellation diagram. 
2) Calculate the maximum probability metrics, which relates the rest of the $a$ priori LLRs $\left\{L_{a}\left(b_{j}\right)\right\}_{j=\mathrm{BPS} / 2+2}^{\mathrm{BPS}}$ and $\left\{L_{a}\left(b_{j}\right)\right\}_{j=2}^{\mathrm{BPS} / 2}$ to the PAM magnitude index $i$ as:

$$
\begin{aligned}
& d_{\mathrm{Re}_{i}}=\left|t_{\mathrm{Re}_{i}}\right|+\sum_{j=\mathrm{BPS} / 2+2}^{\mathrm{BPS}} \widetilde{b}_{j} L_{a}\left(b_{j}\right)-\frac{A_{i}^{2}}{\widetilde{N}_{0}}, \\
& d_{\mathrm{Im}_{i}}=\left|t_{\mathrm{Im}_{i}}\right|+\sum_{j=2}^{\mathrm{BPS} / 2} \widetilde{b}_{j} L_{a}\left(b_{j}\right)-\frac{A_{i}^{2}}{\tilde{N}_{0}} .
\end{aligned}
$$

3) For the first bit and the (BPS/2+1)-th bit, which determine the signs, the a posteriori LLRs are given by:

$$
\begin{aligned}
L_{p}\left(b_{1}\right)= & \max _{i \in\{1, \cdots, \sqrt{L} / 2\}}\left(d_{\operatorname{Im}_{i}}^{b_{1}=1}\right) \\
& -\max _{i \in\{1, \cdots, \sqrt{L} / 2\}}\left(d_{\operatorname{Im}_{i}}^{b_{1}=0}\right), \\
L_{p}\left(b_{\mathrm{BPS} / 2+1}\right)= & \max _{i \in\{1, \cdots, \sqrt{L} / 2\}}\left(d_{\operatorname{Re}_{i} / 2+1}^{b_{\mathrm{BPS} / 2}=1}\right) \\
& -\max _{i \in\{1, \cdots, \sqrt{L} / 2\}}\left(d_{\mathrm{Re}_{i} / 2+1}^{b_{\mathrm{BPS}}=0}\right),
\end{aligned}
$$

where the probability metrics of Eq. (30) have to be updated when the specific bit is set to be 1 or 0 as:

$$
\begin{aligned}
& d_{\operatorname{Im}_{i}}^{b_{1}=1}=-t_{\operatorname{Im}_{i}}+\sum_{j=2}^{\mathrm{BPS} / 2} \widetilde{b}_{j} L_{a}\left(b_{j}\right)-\frac{A_{i}^{2}}{N_{0}}, \\
& d_{\operatorname{Im}_{i}}^{b_{1}=0}=t_{\mathrm{Im}_{i}}+\sum_{j=2}^{\mathrm{BPS} / 2} \widetilde{b}_{j} L_{a}\left(b_{j}\right)-\frac{A_{i}^{2}}{\widetilde{N}_{0}} . \\
& d_{\mathrm{Re}_{i}}^{b_{\mathrm{BPS}^{2}+1}=1}=-t_{\mathrm{Re}_{i}}+\sum_{j=\mathrm{BPS} / 2+2}^{\mathrm{BPS}} \widetilde{b}_{j} L_{a}\left(b_{j}\right)-\frac{A_{i}^{2}}{N_{0}}, \\
& d_{\mathrm{Re}_{i}}^{b_{\mathrm{BP}_{2}+1}=0}=t_{\mathrm{Re}_{i}}+\sum_{j=\mathrm{BPS} / 2+2}^{\mathrm{BPS}} \widetilde{b}_{j} L_{a}\left(b_{j}\right)-\frac{A_{i}^{2}}{\widetilde{N}_{0}} .
\end{aligned}
$$

4) For the rest (BPS - 2) bits, which determine the magnitudes, the Max-Log-MAP algorithm is given by:

$$
\begin{aligned}
L_{p}\left(b_{k}\right)= & \max _{b_{k}=1}\left(d_{\mathrm{Im}_{i}}\right)-\max _{b_{k}=0}\left(d_{\mathrm{Im}_{i}}\right), \\
& k \in\{2, \cdots, \operatorname{BPS} / 2\}, \\
L_{p}\left(b_{k}\right)= & \max _{b_{k}=1}\left(d_{\mathrm{Re}_{i}}\right)-\max _{b_{k}=0}\left(d_{\mathrm{Re}_{i}}\right), \\
& k \in\{\mathrm{BPS} / 2+2, \cdots, \mathrm{BPS}\},
\end{aligned}
$$

where the tentative indices set for $(i \in\{1, \cdots, \sqrt{L} / 2\})$ is halved when a specific bit $b_{k}$ is fixed to 1 or 0 .

The corresponding reduced-complexity Approx-Log-MAP algorithm conceived for Square $L \mathrm{QAM}$ may be obtained by appropriately modifying the proposed Max-Log-MAP algorithm, where the max operation should be replaced by jac operation, while the operation $(|t|)$ should be replaced by the $\Lambda(|t|)$.

In the design of reduced-complexity soft QAM detection algorithms, we aim for a reduced-complexity order of $O\left(\log _{2} L\right)$. However, both the real and imaginary part of a Square $L$ QAM symbol has (BPS/2-1) bits, which encode the PAM magnitude together, hence they have to be jointly detected. As a result, when detecting the (BPS -2$)$ bits which

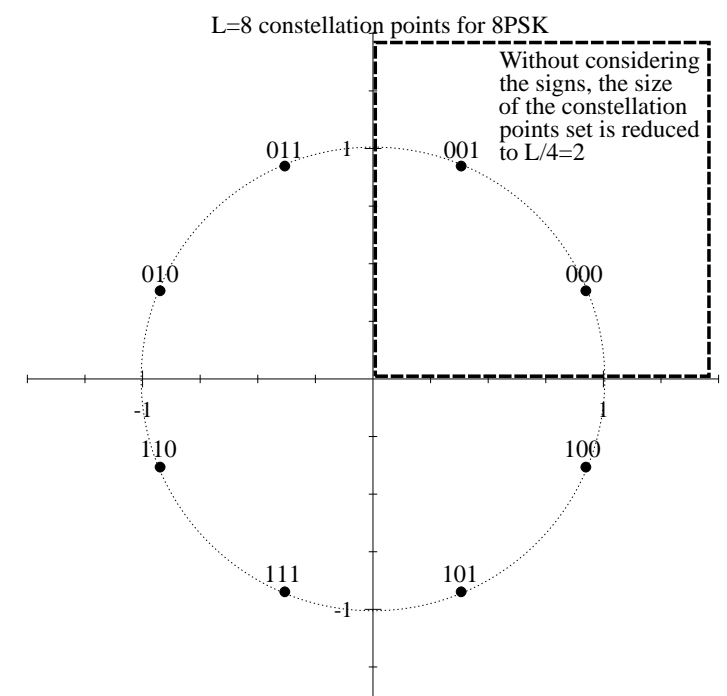

Fig. 2. Constellation diagram of rotated 8PSK. We deliberately rotate all the LPSK ( $L \geq 4$ ) constellation diagrams anti-clockwise by a phase of $\pi / L$, so that there are exactly $L / 4$ constellation points in each quadrant.

determine the magnitudes, the complexity order of Eq. (34) is given by $O(\sqrt{L} / 2)$. Furthermore, when detecting the two bits which determine the signs, the complexity order of Eq. (31) is given by $O(\sqrt{L})$, because the constellation set has to be updated twice, when the specific bit is fixed to 1 and 0 .

\section{Different PSK/QAM Constellations}

For Square $L \mathrm{QAM}$ schemes, the signs and the magnitudes of the real and imaginary parts of a transmitted symbol are encoded separately. By contrast, a high-order LPSK scheme associated with $(L>4)$ encodes its phase, which means that the magnitudes of the real and imaginary parts of a transmitted LPSK symbol are in fact encoded jointly. As a result, the jointly encoded bits in a LPSK symbol have to be detected together, which imposes a higher complexity. However, it can be seen in Algorithm 1 that as long as we have two bits, which determine the signs of the real and imaginary parts respectively, a similar reduced-complexity detection algorithm may be obtained. For the sake of achieving this goal, we have to rotate all the LPSK constellations (except BPSK) in [19] anti-clockwise by $(\pi / L)$, so that there are exactly $L / 4$ constellation points in each quadrant. The rotated 8PSK constellation is shown in Fig. 2.

Similarly, a Star LQAM constellation [22], [23] may be rotated anti-clockwise by a phase angle of $\left(\pi / L_{P}\right)$, where $L_{A}$ and $L_{P}$ refers to the number of constellation rings and the number of phasors, respectively. More explicitly, a Star $L \mathrm{QAM}$ symbol may be represented by $s_{n}=\gamma_{n} \sigma_{n}$, where $\log _{2} L_{A}$ bits are assigned for encoding the radius $\left\{\gamma_{n}^{a}\right\}_{a=1}^{L_{A}}$, while the following $\log _{2} L_{P}$ bits are assigned for encoding the phase $\left\{\sigma_{n}^{p}\right\}_{p=1}^{L_{P}}$. If we define the ring ratio of a Star $L \mathrm{QAM}$ symbol as the ratio of the ring radii, we have $\beta=\left\{\frac{\gamma^{(p+1)}}{\gamma^{p}}\right\}_{p=1}^{L_{A}-1}$. Then the symbol power normalization factor is given by $\alpha=$ $\frac{\sum_{p=1}^{L_{A}} \beta^{2(p-1)}}{L_{A}}$. Therefore, the Star LQAM ring radius should be normalized as $\left\{\gamma^{p}=\frac{\beta^{(p-1)}}{\sqrt{\alpha}}\right\}_{p=1}^{L_{A}}$. It was shown in [22], [23] 
that the two-ring Star 16/32QAM associated with $(\beta=2.0)$ and the four-ring Star 64QAM associated with $(\beta=1.4)$ have the optimum performance in Rayleigh fading channels.

In order to operate the Max-Log-MAP algorithm conceived for different PSK/QAM constellations at a reduced complexity, we extend the a posteriori probability metric of Eq. (19) as:

$$
\begin{aligned}
d_{l}= & -\frac{\left|\widetilde{z}_{n}\right|^{2}}{\widetilde{N}_{0}}-\frac{\left|s^{l}\right|^{2}}{\widetilde{N}_{0}} \\
& +\frac{\operatorname{Re}\left(\widetilde{z}_{n}\right) \operatorname{Re}\left(s^{l}\right)+\operatorname{Im}\left(\widetilde{z}_{n}\right) \operatorname{Im}\left(s^{l}\right)}{\bar{N}_{0}}+\sum_{j=1}^{\mathrm{BPS}} \widetilde{b}_{j} L_{a}\left(b_{j}\right),
\end{aligned}
$$

where the constant of $\left(-\frac{\left|\widetilde{z}_{n}\right|^{2}}{\tilde{N}_{0}}\right)$ may be ignored. Let us now consider the 8PSK constellation of Fig. 2 as an example, where the eight metrics $\left\{d_{l}\right\}_{l=1}^{8}$ of Eq. (35) may be expressed as:

$$
\begin{gathered}
d_{1}=t_{\mathrm{Re} 1}+t_{\mathrm{Im} 1}+C_{8 \mathrm{PSK}}, \quad d_{3}=-t_{\mathrm{Re} 1}+t_{\mathrm{Im} 1}+C_{8 \mathrm{PSK}} \\
d_{5}=t_{\mathrm{Re} 1}-t_{\mathrm{Im} 1}+C_{8 \mathrm{PSK}}, \quad d_{7}=-t_{\mathrm{Re} 1}-t_{\mathrm{Im} 1}+C_{8 \mathrm{PSK}} \\
d_{2}=t_{\mathrm{Re} 2}+t_{\mathrm{Im} 2}+L_{a}\left(b_{3}\right)+C_{8 \mathrm{PSK}} \\
d_{4}=-t_{\mathrm{Re} 2}+t_{\mathrm{Im} 2}+L_{a}\left(b_{3}\right)+C_{8 \mathrm{PSK}} \\
d_{6}=t_{\mathrm{Re} 2}-t_{\mathrm{Im} 2}+L_{a}\left(b_{3}\right)+C_{8 \mathrm{PSK}} \\
d_{8}=-t_{\mathrm{Re} 2}-t_{\mathrm{Im} 2}+L_{a}\left(b_{3}\right)+C_{8 \mathrm{PSK}}
\end{gathered}
$$

where we relate the real and imaginary parts of $\widetilde{z}_{n}$ to the corresponding soft bit input $L_{a}\left(b_{2}\right)$ and $L_{a}\left(b_{1}\right)$ as:

$$
\begin{array}{ll}
t_{\operatorname{Re} 1}=\frac{\cos \left(\frac{\pi}{8}\right) \operatorname{Re}\left(\widetilde{z}_{n}\right)}{\bar{N}_{0}}-\frac{L_{a}\left(b_{2}\right)}{2}, & t_{\operatorname{Re} 2}=\frac{\sin \left(\frac{\pi}{8}\right) \operatorname{Re}\left(\widetilde{z}_{n}\right)}{\bar{N}_{0}}-\frac{L_{a}\left(b_{2}\right)}{2}, \\
t_{\operatorname{Im} 1}=\frac{\sin \left(\frac{\pi}{8}\right) \operatorname{Im}\left(\widetilde{z}_{n}\right)}{\bar{N}_{0}}-\frac{L_{a}\left(b_{1}\right)}{2}, & t_{\operatorname{Im} 2}=\frac{\cos \left(\frac{\pi}{8}\right) \operatorname{Im}\left(\widetilde{z}_{n}\right)}{\bar{N}_{0}}-\frac{L_{a}\left(b_{1}\right)}{2},
\end{array}
$$

while the constant $C_{8 \mathrm{PSK}}$ is given by $\left[C_{\text {8PSK }}=-\frac{1}{\widetilde{N}_{0}}+\frac{L_{a}\left(b_{1}\right)+L_{a}\left(b_{2}\right)}{2}\right]$. The eight probability metrics $\left\{d_{l}\right\}_{l=1}^{8}$ are arranged into two groups in Eqs. (36) and (37), where every four metrics are associated with the same constellation magnitudes. It can be seen that the four metrics formulated in Eq. (36) all contain three parts, i.e. they are $\pm t_{\mathrm{Re} 1}, \pm t_{\mathrm{Im} 1}$ and $C_{8 \mathrm{PSK}}$. As a result, the maximum metric over the four candidates in Eq. (36) is given by $\max _{l=\{1,3,5,7\}} \quad d_{l}=\left|t_{\mathrm{Re} 1}\right|+\left|t_{\mathrm{Im} 1}\right|+C_{8 \mathrm{PSK}}$. Similarly, the maximum metric over the second group in Eq. (37) is given by $\max _{l=\{2,4,6,8\}} \quad d_{l}=\left|t_{\mathrm{Re} 2}\right|+\left|t_{\mathrm{Im} 2}\right|+L_{a}\left(b_{3}\right)+C_{8 \mathrm{PSK}}$. Therefore, the maximum a posteriori probability metric generated by the Max-Log-MAP algorithm is given by:

$$
d_{\max }=\max \left\{\begin{array}{l}
\left|t_{\mathrm{Re} 1}\right|+\left|t_{\mathrm{Im} 1}\right|+C_{8 \mathrm{PSK}} \\
\left|t_{\mathrm{Re} 2}\right|+\left|t_{\mathrm{Im} 2}\right|+L_{a}\left(b_{3}\right)+C_{8 \mathrm{PSK}}
\end{array}\right\} \text {. }
$$

Instead of evaluating and comparing Eq. (35) eight times in Eqs. (36) and (37), Eq. (39) only has to evaluate and compare two candidates in order to obtain $d_{\max }$. In other words, $d_{\max }$ is obtained without visiting all the eight 8PSK constellation points. In fact, only the two constellation points in the first quadrant are of interest, as demonstrated by Fig. 2. In more detail, the reduced-complexity Max-Log-MAP algorithm may be formulated as:

$$
\begin{aligned}
& L_{p}\left(b_{1}\right)=d_{\max }^{b_{1}=1}-d_{\max }^{b_{1}=0} \\
& L_{p}\left(b_{2}\right)=d_{\max }^{b_{2}=1}-d_{\max }^{b_{2}=0} \\
& L_{p}\left(b_{3}\right)=\left|t_{\mathrm{Re} 2}\right|+\left|t_{\operatorname{Im} 2}\right|+L_{a}\left(b_{3}\right)-\left|t_{\operatorname{Re} 1}\right|-\left|t_{\operatorname{Im} 1}\right|,
\end{aligned}
$$

where $d_{\max }^{b_{1}=1}$ and $d_{\max }^{b_{1}=0}$ may be obtained by replacing $\left\{\left|t_{\operatorname{Im} i}\right|\right\}_{i=1}^{2}$ in Eq. (39) by $\left\{-t_{\operatorname{Im} i}\right\}_{i=1}^{2}$ and $\left\{t_{\operatorname{Im} i}\right\}_{i=1}^{2}$, respectively, while $d_{\max }^{b_{2}=1}$ and $d_{\max }^{b_{2}=0}$ are obtained by replacing $\left\{\left|t_{\mathrm{Re} i}\right|\right\}_{i=1}^{2}$ in Eq. (39) by $\left\{-t_{\mathrm{Re} i}\right\}_{i=1}^{2}$ and $\left\{t_{\mathrm{Re} i}\right\}_{i=1}^{2}$, respectively. The constant $C_{8 \mathrm{PSK}}$ in Eq. (39) may be omitted.

Based on the example of 8PSK detection, we propose the reduced-complexity Max-Log-MAP algorithm conceived for general $L \mathrm{PSK} / \mathrm{QAM}$ Detection as follows:

\section{Algorithm 2: Max-Log-MAP Algorithm for General} LPSK/QAM Detection.

1) Define the test-variables, which relates the first two a priori LLRs $L_{a}\left(b_{2}\right)$ and $L_{a}\left(b_{1}\right)$ to the real and imaginary parts of $\widetilde{z}_{n}$ as:

$$
\begin{aligned}
t_{\mathrm{Re}_{i}} & =\frac{A_{i} \operatorname{Re}\left(\widetilde{z}_{n}\right)}{\bar{N}}-\frac{L_{a}\left(b_{2}\right)}{2}, \\
t_{\mathrm{Im}_{i}} & =\frac{B_{i} \mathrm{Im}\left(\widetilde{z}_{n}\right)}{\bar{N}_{0}}-\frac{L_{a}\left(b_{1}\right)}{2},
\end{aligned}
$$

where $\left\{\left(A_{i}, B_{i}\right)\right\}_{i=1}^{L / 4}$ denote the coordinates of the LPSK/QAM constellation points which are located in the first quadrant.

2) Calculate the maximum probability metrics, which relates the rest of the a priori LLRs $\left\{L_{a}\left(b_{j}\right)\right\}_{j=3}^{\mathrm{BPS}}$ to the magnitude index $i$ as:

$$
d_{i}=\left|t_{\mathrm{Re}_{i}}\right|+\left|t_{\mathrm{Im}_{i}}\right|+\sum_{j=3}^{\mathrm{BPS}} \widetilde{b}_{j} L_{a}\left(b_{j}\right)-\frac{A_{i}^{2}+B_{i}^{2}}{\widetilde{N}_{0}} .
$$

3) For the first two bits, which determine the signs, the $a$ posteriori LLRs are given by:

$$
\begin{aligned}
L_{p}\left(b_{1}\right)= & \max _{i \in\{1, \cdots, L / 4\}}\left(d_{i}^{b_{1}=1}\right) \\
& -\max _{i \in\{1, \cdots, L / 4\}}\left(d_{i}^{b_{1}=0}\right), \\
L_{p}\left(b_{2}\right)= & \max _{i \in\{1, \cdots, L / 4\}}\left(d_{i}^{b_{2}=1}\right) \\
& -\max _{i \in\{1, \cdots, L / 4\}}\left(d_{i}^{b_{2}=0}\right),
\end{aligned}
$$

where the probability metrics of Eq. (42) have to be updated as:

$$
\begin{aligned}
& d_{i}^{b_{1}=1}=\left|t_{\mathrm{Re}_{i}}\right|-t_{\mathrm{Im}_{i}}+\sum_{j=3}^{\mathrm{BPS}} \widetilde{b}_{j} L_{a}\left(b_{j}\right)-\frac{A_{i}^{2}+B_{i}^{2}}{\widetilde{N}_{0}}, \\
& d_{i}^{b_{1}=0}=\left|t_{\mathrm{Re}_{i}}\right|+t_{\mathrm{Im}_{i}}+\sum_{j=3}^{\mathrm{BPS}} \widetilde{b}_{j} L_{a}\left(b_{j}\right)-\frac{A_{i}^{2}+B_{i}^{2}}{\widetilde{N}_{0}} . \\
& d_{i}^{b_{2}=1}=-t_{\mathrm{Re}_{i}}+\left|t_{\mathrm{Im}_{i}}\right|+\sum_{j=3}^{\mathrm{BPS}} \widetilde{b}_{j} L_{a}\left(b_{j}\right)-\frac{A_{i}^{2}+B_{i}^{2}}{\widetilde{N}_{0}}, \\
& d_{i}^{b_{2}=0}=t_{\mathrm{Re}_{i}}+\left|t_{\mathrm{Im}_{i}}\right|+\sum_{j=3}^{\mathrm{BPS}} \widetilde{b}_{j} L_{a}\left(b_{j}\right)-\frac{A_{i}^{2}+B_{i}^{2}}{\widetilde{N}_{0}} .
\end{aligned}
$$

4) For the following $(\mathrm{BPS}-2)$ bits which determine the magnitudes, the Max-Log-MAP algorithm is given by:

$$
\begin{gathered}
L_{p}\left(b_{k}\right)=\max _{b_{k}=1}\left(d_{i}\right)-\max _{b_{k}=0}\left(d_{i}\right), \\
k \in\{3, \cdots, \text { BPS }\},
\end{gathered}
$$

where the tentative index set for $(i \in\{1, \cdots, L / 4\})$ is halved, when a specific bit $b_{k}$ is fixed to 1 or 0 .

The corresponding reduced-complexity Approx-Log-MAP 
algorithm may be obtained by replacing the max operation by the jac operation, and by replacing the operation $|t|$ by the $\Lambda(|t|)$.

When detecting the two bits, which determine the signs, Eq. (43) in Algorithm 2 has a detection complexity order of $O(L / 2)$, while Eq. (46) evaluated for detecting those specific (BPS-2) bits, which determine the magnitudes has a detection complexity order of $O(L / 4)$. They exhibit a substantially reduced complexity compared to $O(L)$, owing to the fact that the proposed detection algorithms visit a reduced-size fraction of the constellation points.

In summary, Algorithm 1 introduced in Sec. III-B is conceived for Square LQAM detection, while Algorithm 2 introduced in Sec. III-C is conceived for detecting general LPSK/QAM constellations. We note that when Algorithm 2 is invoked for $L$ PSK detection, the constant $\left(\frac{A_{i}^{2}+B_{i}^{2}}{\widetilde{N}_{0}}=\frac{1}{\widetilde{N}_{0}}\right)$ in Eqs. (42), (44) and (45) may be ignored. Furthermore, when the number of bits per Square LQAM symbol is an odd number, Algorithm 1 may be readily modified, where the real positive PAM magnitudes have $\sqrt{2 L} / 2$ candidates, while the imaginary positive PAM magnitudes have $\sqrt{L / 2} / 2$ candidates. Furthermore, it was shown in [24] that Cross LQAM constellations actually have a better performance compared to Square $L$ QAM schemes. We note that Algorithm 2 may be adopted for detecting Cross LQAM constellations.

\section{Performance Results}

We discuss our simulation results in this section. We quantify the complexity in terms of the total number of real-valued calculations required for producing a single soft bit output. The complexity reduction achieved by Algorithms 1 and 2 is portrayed in Fig. 3(a) and Fig. 3(b), respectively. In contrast to the Square LQAM results of Fig. 3(a), Algorithm 2 conceived for general $L P S K / Q A M$ detection achieves a higher complexity reduction, as demonstrated in Fig. 3(b). This is because the conventional Square LQAM detection presented in Sec. III-A already has a relatively low detection complexity, owing to the fact that the real and imaginary parts of a Square $L \mathrm{QAM}$ symbol are detected separately. Nonetheless, the complexity reduction seen in both Fig. 3(a) and Fig. 3(b) is substantial especially, when the soft PSK/QAM detector is invoked several times in turbo detection applications. Furthermore, as the number of modulation levels $L$ increases, the complexity reduction becomes even more substantial, which is evidenced by Figs. 3(a) and 3(b).

The EXIT chart [5] of the reduced-complexity Square $L$ QAM detectors invoked by the MIMO receivers is presented in Fig. 4. Since Alamouti's G2 STBC does not impose any correlation, Fig. 4(a) shows that the Square 16QAM detector has a near-horizontal EXIT curve. However, as the number of modulation levels $L$ increases, Square $L$-QAM constellations involve more bits in jointly encoding the symbol magnitudes, which results in an improved iteration gain, as evidenced by Fig. 4(a). Moreover, Fig. 4(b) demonstrates that the V-BLAST MMSE detector further improves the attainable iteration gain, while performance loss is imposed, when the QAM detectors do not take into account the a priori LLRs, as in the conventional bit metric generation methods presented in [13]-[15].

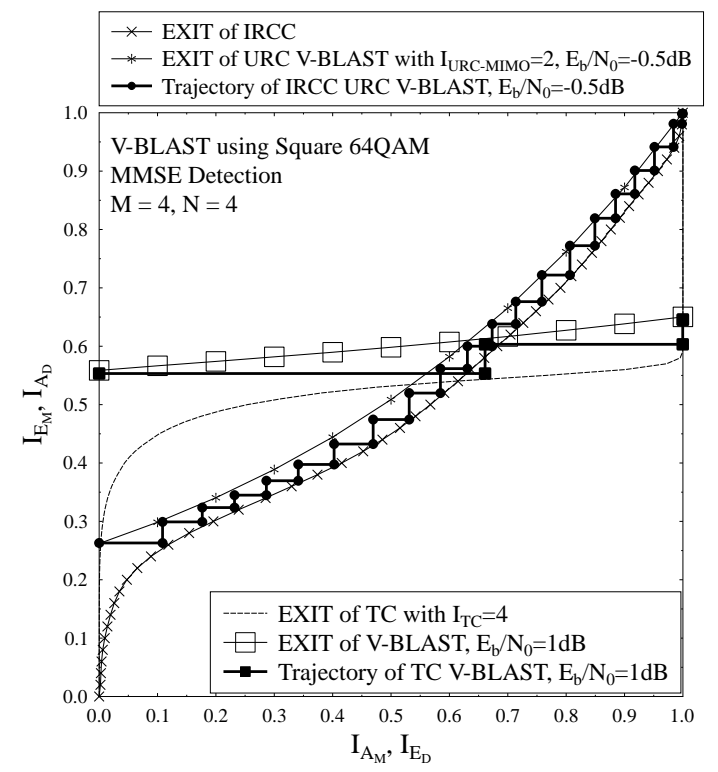

Fig. 5. Decoding trajectory recorded for TC V-BLAST and IRCC URC V-BLAST. The reduced-complexity Max-Log-MAP algorithm is employed.

In order to demonstrate the benefit of the soft QAM detectors' iteration gain, we also applied our detection algorithms in channel-coded systems. The half-rate TC employed is constituted by two half-rate Recursive Convolutional Codes (RSCs) associated with a constraint length of $K=3$ (a octal generator polynomial of $[7,5]$ ) and with the half-rate puncturing of the parity bits, while the schematic of the IRCC-URC MIMO system may be found in [25]. We portray the resultant MonteCarlo simulation based decoding trajectory in Fig. 5. The BER performance of channel-coded STBC systems is portrayed in Fig. 6(a), which shows that a significant performance improvement is achieved, when the number of outer iterations is increased ( $\left.I_{\text {out }}=\{1,2,3\}\right)$, when four inner TC iterations $\left(I_{T C}=4\right)$ are used. Nonetheless, Fig. 6(a) shows that a similar performance may be obtained by employing a stronger TC associated with $\left(I_{T C}=12\right)$ and $\left(I_{\text {out }}=1\right)$, while an even better performance is recorded when the IRCC and URC schemes of [25] are applied. However, Fig. 6(a) demonstrates that a significantly improved near-capacity performance is achieved upon increasing the number of inner iterations to $I_{U R C-M I M O}=2$, which would be simply impossible without considering the a priori LLRs, when a high-throughput QAM detector is invoked. Moreover, the performance of the VBLAST MMSE system seen in Fig. 6(b) further confirms the performance advantage of our proposed QAM detection algorithms in the context of different channel-coded scenarios. The maximum achievable rate indicated in Fig. 6 is calculated based on the area property of the EXIT chart [5].

The non-Square QAM schemes [22]-[24] are also widely used in many communication systems. As demonstrated by the EXIT charts and BER curves of channel-coded STBC using 32QAM in Figs. 7 and 8, Cross 32QAM and Star 32QAM outperform their Square 32QAM counterpart. However, the conventional Square LQAM detection invoking Eqs. (20) exhibits a substantially lower detection complexity compared to non-Square $L$ QAM detection relying on Eq. (19), as demon- 


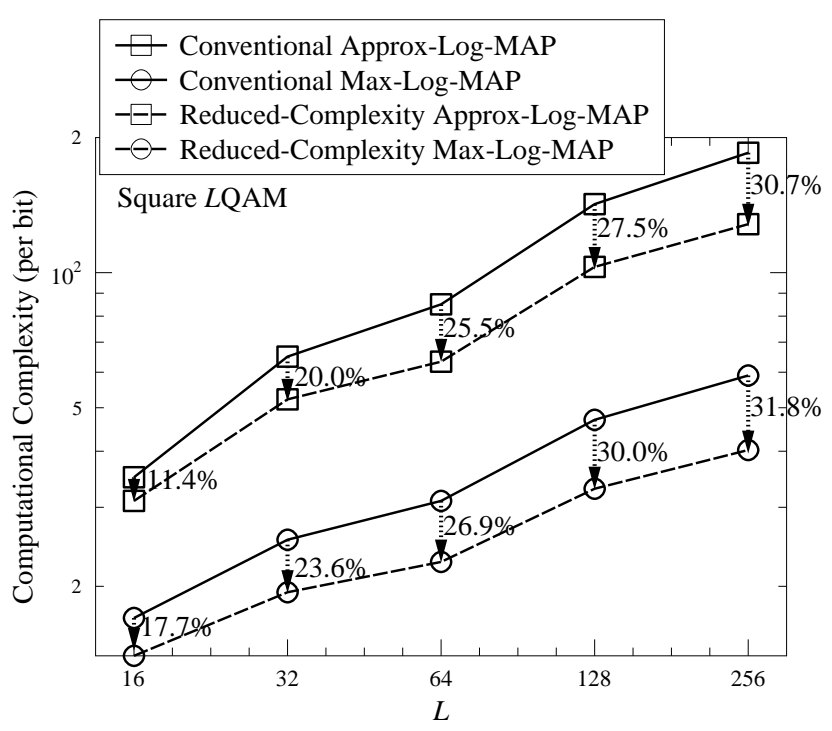

(a) Square $L \mathrm{QAM}$.

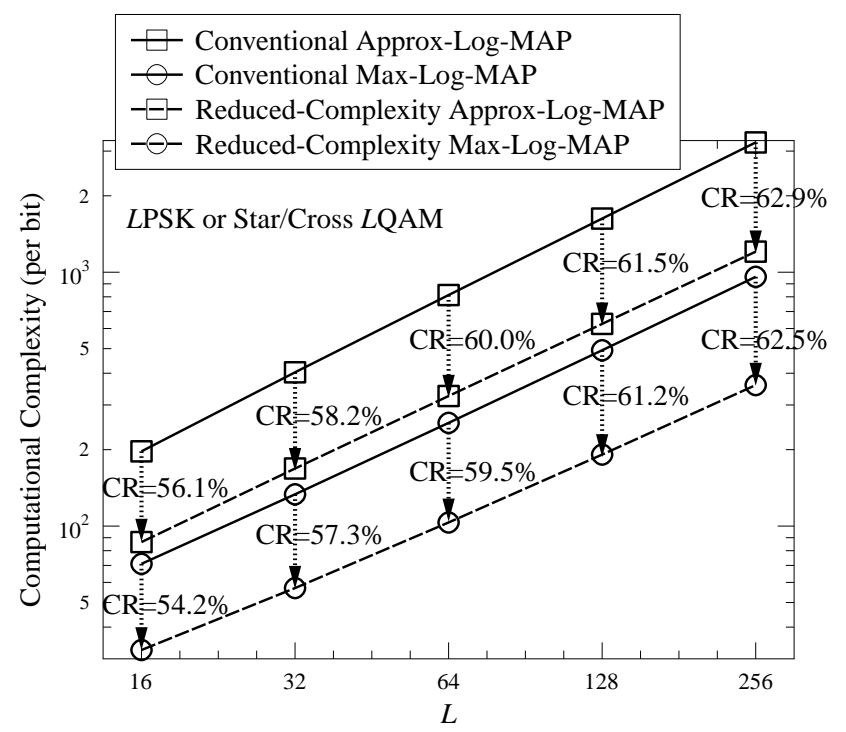

(b) LPSK or Star/Cross LQAM.

Fig. 3. Complexity comparison between the conventional soft LPSK/QAM detection algorithms and the reduced-complexity detection algorithms. The complexity reduction achieved by the proposed detection algorithms is indicated on the figures.

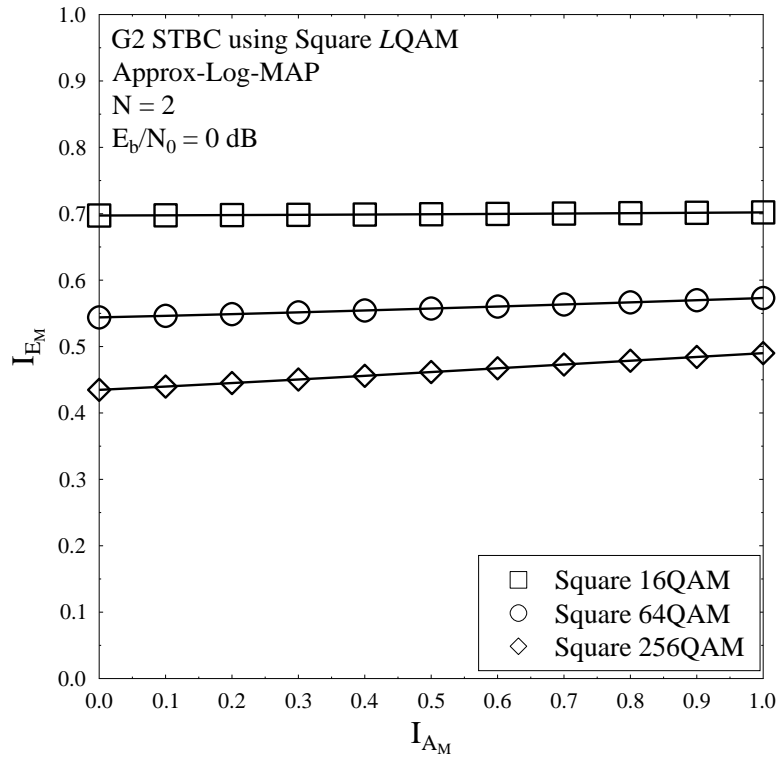

(a) $\mathrm{G} 2 \operatorname{STBC}(N=2)$.

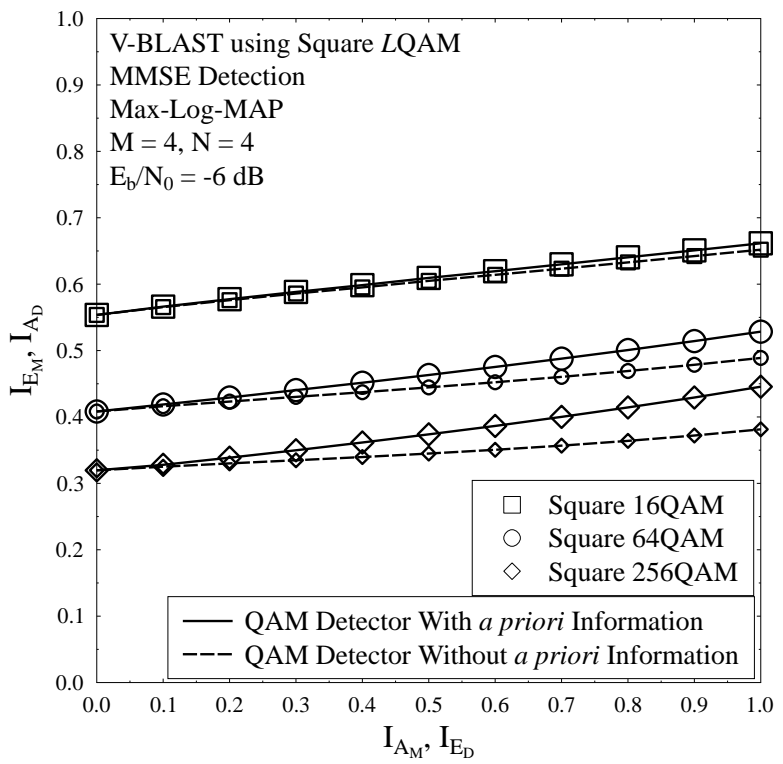

(b) V-BLAST $(M=4, N=4)$.

Fig. 4. EXIT charts of the reduced-complexity detection algorithms when they are invoked to detect G2 STBC as well as the MMSE aided V-BLAST.

strated by Fig. 9. Nonetheless, it can also be seen in Fig. 9 that the complexity difference between the Cross/Star 32QAM detection and the Square 32QAM detection is significantly reduced when the proposed reduced-complexity detection algorithms are applied.

\section{Discussions}

The proposed Algorithms 1 and 2 achieve their substantial complexity reduction by exploiting the symmetry provided by each single Gray-labelled constellation diagram. This philoso- phy cannot be directly applied to ML V-BLAST MIMO detection invoking Eq. (3), since there is no inherent symmetry between the different MIMO links' output signal constellations, as they are faded completely independently. Furthermore, the non-linear Sphere Decoder (SD) conceived for V-BLAST in [26] also cannot invoke our proposed algorithm, because the constellation's symmetry is ignored, when we only consider the constellation points falling within the SD's search radius, which is explicitly shown in Figs. 2 and 3 of [26].

Nonetheless, the reduced-complexity PSK/QAM detectors 


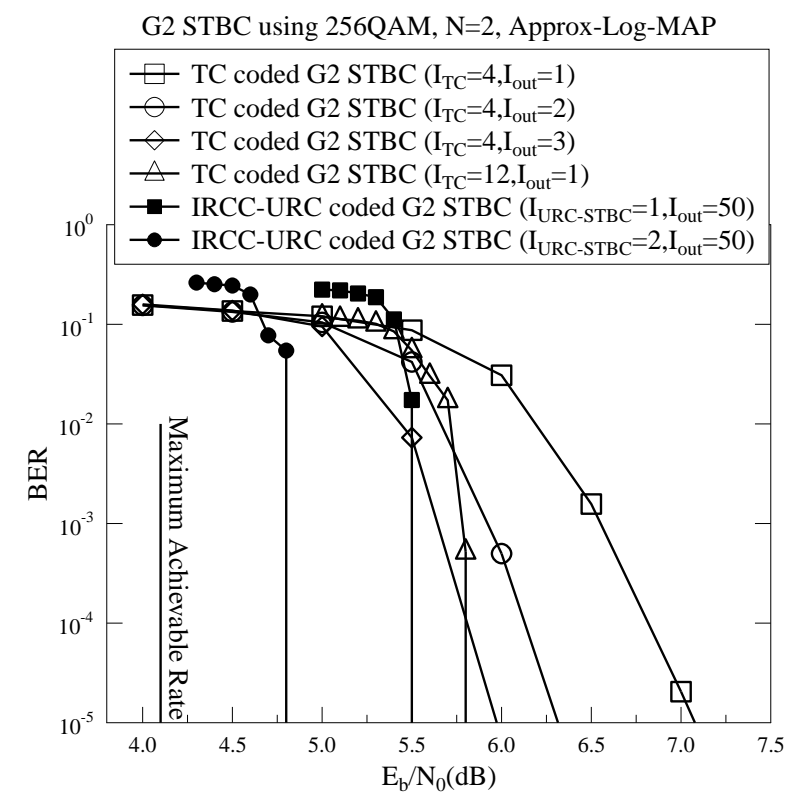

(a) $\mathrm{G} 2 \operatorname{STBC}(N=2)$.

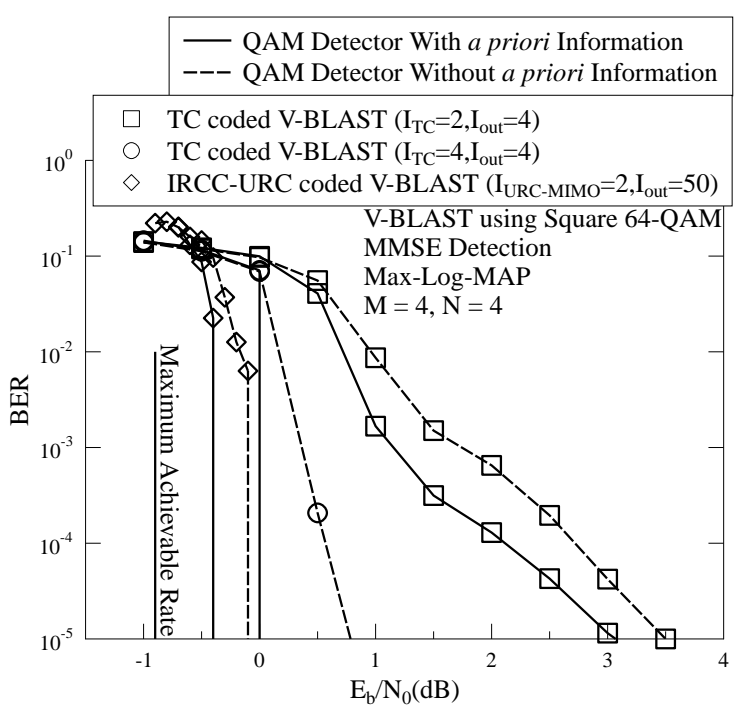

(b) V-BLAST $(M=4, N=4)$.

Fig. 6. BER performance of the TC/IRCC-URC MIMO systems with turbo detection.

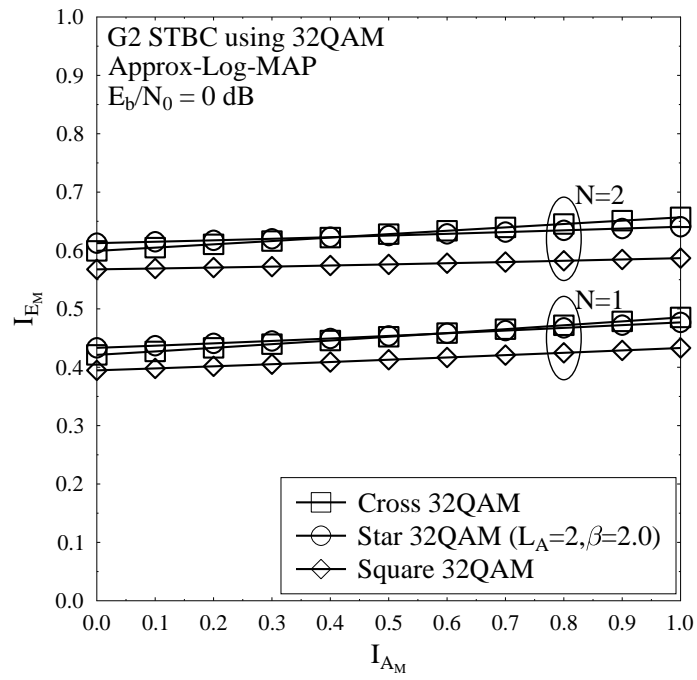

Fig. 7. EXIT charts of $\mathrm{G} 2 \mathrm{STBC}$ using different 32QAM constellations.

proposed in this paper may benefit a diverse variety of communication systems. As demonstrated in Sec. II, the linear MIMO receivers as well as orthogonal STBC detectors may directly invoke our proposed PSK/QAM detection algorithms. Furthermore, linear receivers designed for beamforming [27] or for CDMA Multiple-User Detection (MUD) [8] have a similar form to the MMSE aided V-BLAST system introduced in Sec. II-A. Hence our reduced-complexity PSK/QAM detection algorithms may also be employed by the aforementioned systems.

\section{Conclusions}

A new method of reducing the complexity of the soft PSK/QAM detection algorithms was proposed, and its advantages in V-BLAST system and in Alamouti's G2 STBC

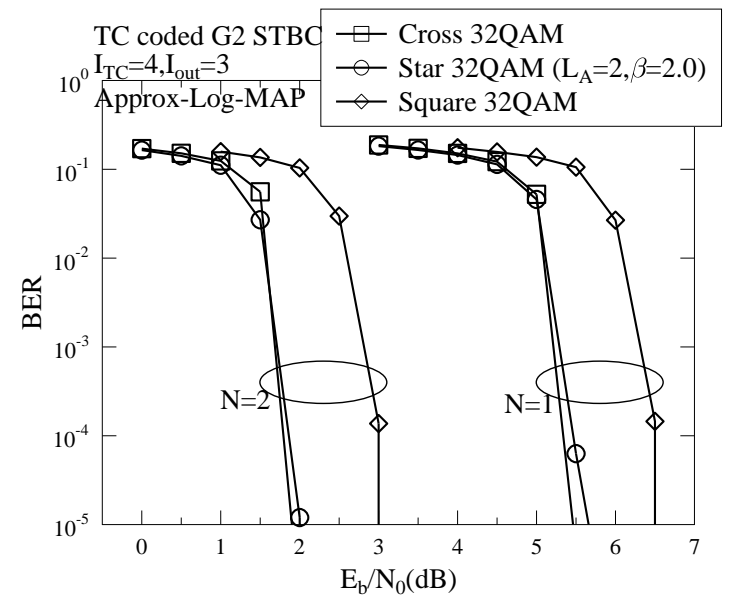

Fig. 8. BER performance of TC G2 STBC using different 32QAM constellations. The number of inner iterations within the TC is set to $I_{T C}=4$, while the number of outer iterations between TC and MIMO is set to $I_{\text {out }}=3$.

system were demonstrated. Our simulation results confirmed that a substantial complexity reduction was achieved both by the reduced-complexity Approx-Log-MAP algorithm as well as by the reduced-complexity Max-Log-MAP algorithm, while no performance degradation was imposed.

\section{ACKNOWLEDGMENT}

The authors would like to express their gratitude to Mr. Shaoshi Yang, Dr. Rong Zhang, Dr. Rob Maunder, Mr. Kent TK Cheung, Mr. Xin Zuo and Mr. Chen Dong for their constructive comments and suggestions.

\section{REFERENCES}

[1] C. Berrou and A. Glavieux, "Near optimum error correcting coding and decoding: Turbo-codes," IEEE Transactions on Communications, vol. 44, pp. $1261-1271$, Oct. 1996. 


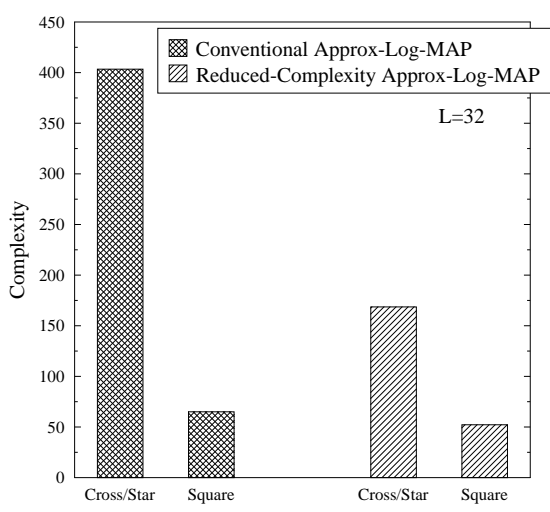

Fig. 9. Complexity comparison between Square 32QAM detection and Cross/Star 32QAM detection.

[2] S. Ten Brink, J. Speidel, and R.-H. Han, "Iterative demapping for QPSK modulation," Electronics Letters, vol. 34, pp. 1459 -1460, July 1998.

[3] S. Ten Brink, J. Speidel, and R.-H. Yan, "Iterative demapping and decoding for multilevel modulation," in IEEE Global Telecommunications Conference (GLOBECOM'98), vol. 1, pp. 579-584, Nov. 1998.

[4] H. Tullberg and P. Siegel, "Serial concatenated TCM with an inner accumulate code-Part I: maximum-likelihood analysis," IEEE Transactions on Communications, vol. 53, pp. 64 - 73, Jan. 2005.

[5] M. Tuchler, "Design of serially concatenated systems depending on the block length," IEEE Transactions on Communications, vol. 52, pp. 209 - 218, Feb. 2004.

[6] L. Kong, S. Ng, R. Tee, R. Maunder, and L. Hanzo, "Reducedcomplexity near-capacity downlink iteratively decoded generalized multi-layer space-time coding using irregular convolutional codes," IEEE Transactions on Wireless Communications, vol. 9, pp. $684-695$, Feb. 2010.

[7] A. Paulraj, D. Gore, R. Nabar, and H. Bolcskei, "An overview of MIMO communications - a key to gigabit wireless," Proceedings of the IEEE, vol. 92, pp. $198-218$, Feb. 2004.

[8] X. Wang and H. Poor, "Iterative (turbo) soft interference cancellation and decoding for coded CDMA," IEEE Transactions on Communications, vol. 47, pp. $1046-1061$, July 1999.

[9] M. Sellathurai and S. Haykin, "Turbo-BLAST for wireless communications: theory and experiments," IEEE Transactions on Signal Processing, vol. 50, pp. 2538 - 2546, Oct. 2002.

[10] C. J. Foschini, "Layered space-time architecture for wireless communication in a fading environment when using multiple antennas," Bell Labs. Tech. J., vol. 1, no. 2, pp. 41-59, 1996.

[11] S. Alamouti, "A simple transmit diversity technique for wireless communications," IEEE Journal on Selected Areas in Communications, vol. 16, pp. $1451-1458$, Oct. 1998.

[12] V. Tarokh, H. Jafarkhani, and A. Calderbank, "Space-time block codes from orthogonal designs," IEEE Transactions on Information Theory, vol. 45, pp. 1456 -1467, July 1999.

[13] F. Tosato and P. Bisaglia, "Simplified soft-output demapper for binary interleaved COFDM with application to HIPERLAN/2," in IEEE International Conference on Communications, 2002 (ICC 2002), vol. 2, pp. $664-668$ vol.2, 2002.

[14] K. Hyun and D. Yoon, "Bit metric generation for gray coded QAM signals," IEE Proceedings Communications, vol. 152, pp. 1134 - 1138, Dec. 2005.

[15] L. Wang, D. Xu, and X. Zhang, "Low complexity bit metric generation for PAM signals based on nonlinear function," Electronics Letters, vol. 47, pp. 966 -967, Aug. 2011.

[16] ITU-R, "Coexistence between IMT-2000 PCDMA-DS and IMT-2000 OFDMA TDD WMAN in the $2500-2690 \mathrm{mhz}$ band operating in adjacent bands in the same area." [Online]. Available: http://www.itu. int/pub/R-REP-M.2146-2009, 2009.

[17] Qualcomm, "IEEE802.11ac: The next evolution of Wi-Fi standards." [Online]. Available: http://www.qualcomm.com/media/documents/files/ ieee802-11ac-the-next-evolution-of-wi-fi.pdf, 2012.

[18] W. Koch and A. Baier, "Optimum and sub-optimum detection of coded data disturbed by time-varying intersymbol interference," in IEEE
Global Telecommunications Conference (GLOBECOM'90), pp. 1679 1684 vol.3, Dec. 1990

[19] L. Hanzo, S. X. Ng, W. T. Webb, and T. Keller, Quadrature Amplitude Modulation: From Basics to Adaptive Trellis-Coded, Turbo-Equalised and Space-Time Coded OFDM, CDMA and MC-CDMA Systems, 3rd Edition. John Wiley \& Sons, 2004.

[20] P. Robertson, E. Villebrun, and P. Hoeher, "A comparison of optimal and sub-optimal MAP decoding algorithms operating in the log domain," in IEEE International Conference on Communications (ICC'95), June 1995.

[21] L. Hanzo, T. Liew, B. Yeap, R. Tee, and S. Ng, Turbo Coding, Turbo Equalisation and Space-Time Coding (EXIT-Chart-Aided Near-Capacity Designs for Wireless Channels). John Wiley \& Sons, 2011.

[22] X. Dong, N. Beaulieu, and P. Wittke, "Error probabilities of twodimensional $M$-ary signaling in fading," IEEE Transactions on Communications, vol. 47, pp. 352 -355, Mar. 1999.

[23] T. May, H. Rohling, and V. Engels, "Performance analysis of Viterbi decoding for 64-DAPSK and 64-QAM modulated OFDM signals," IEEE Transactions on Communications, vol. 46, pp. 182 -190, Feb. 1998.

[24] P. Vitthaladevuni, M.-S. Alouini, and J. Kieffer, "Exact BER computation for cross QAM constellations," IEEE Transactions on Wireless Communications, vol. 4, pp. 3039 - 3050, Nov. 2005.

[25] C. Xu, S. X. Ng, and L. Hanzo, "Near-capacity irregular convolutional coded cooperative differential linear dispersion codes using multiplesymbol differential detection," IEEE Signal Processing Letters, vol. 18, pp. $173-176$, Mar. 2011.

[26] B. Hochwald and S. ten Brink, "Achieving near-capacity on a multipleantenna channel," IEEE Transactions on Communications, vol. 51, pp. 389 - 399, Mar. 2003.

[27] S. Tan, S. Chen, and L. Hanzo, "On multi-user EXIT chart analysis aided turbo-detected MBER beamformer designs," IEEE Transactions on Wireless Communications, vol. 7, pp. 314 -323, Jan. 2008.

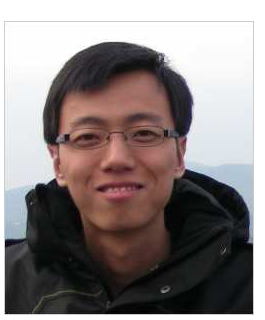

Chao Xu (S'09) received a B.Eng. degree from Beijing University of Posts and Telecommunications, Beijing, China, and a BSc(Eng) with First Class Honours from Queen Mary, University of London, London, UK, through a Sino-UK joint degree program in 2008, both in Telecommunications Enginneering with Management. In 2009, he obtained a MSc degree with distinction in radio frequency communication systems from the University of Southampton, Southampton, UK, and he was awarded IEEE Communications Society UK\&RI Chapter Best MSc Student in Broadband and Mobile Communication Networks. He is currently working towards the $\mathrm{PhD}$ degree with the Research Group of Communications, Signal Processing and Control, School of Electronics and Computer Science, University of Southampton, UK. His research interests include reduced-complexity MIMO design, non-coherent space-time modulation detection, EXIT-chart-aided turbo detection as well as cooperative communications.

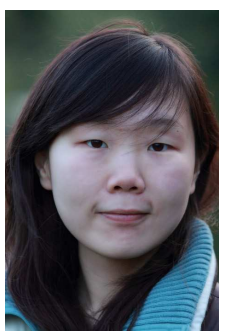

Dandan Liang received her B.Eng. degree (First class) in electronic science and technology from the PLA Information Engineering University, Zhengzhou, China, in 2008 and M.Sc. degree (First class) in radio frequency communication systems from the University of Southampton, UK, in 2009. She is currently working towards the $\mathrm{PhD}$ degree with the Research Group of Communications, Signal Processing and Control, School of Electronics and Computer Science, University of Southampton, UK. Her research interests include adaptive coded modulation, coded modulation, non/coherent modulation detection, iterative detection, networking coding, cooperative communications as well as wirelessoptical fiber communications. 


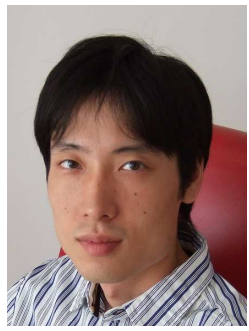

Shinya Sugiura (M'06-SM'12) received the B.S. and M.S. degrees in aeronautics and astronautics from Kyoto University, Kyoto, Japan, in 2002 and 2004, respectively, and the Ph.D. degree in mobile communications from the University of Southampton, Southampton, UK, in 2010. Since 2004, he has been with Toyota Central R\&D Laboratories, Inc., Aichi, Japan, where his research has covered a range of areas in wireless communications, networking, signal processing, and antenna design. He authored/coauthored more than 50 refereed research publications, including 26 IEEE journal and magazine papers. Dr. Sugiura has received a number of distinctions, most recently the 2011 IEEE Communications Society Asia-Pacific Outstanding Young Researcher Award, the 2011 Ericsson Young Scientist Award, and the 2008 IEEE Antennas and Propagation Society Japan Chapter Young Engineer Award.

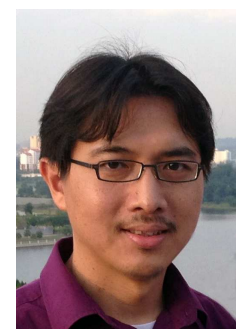

Dr Soon Xin Ng (S'99-M'03-SM'08) received the B.Eng. degree (First class) in electronics engineering and the Ph.D. degree in wireless communications from the University of Southampton, Southampton, U.K., in 1999 and 2002, respectively. From 2003 to 2006, he was a postdoctoral research fellow working on collaborative European research projects known as SCOUT, NEWCOM and PHOENIX. Since August 2006, he has been a member of academic staff in the School of Electronics and Computer Science, University of Southampton. He is involved in the OPTIMIX and CONCERTO European projects as well as the IU-ATC and UC4G projects. He is currently a senior lecturer at the University of Southampton.

His research interests include adaptive coded modulation, coded modulation, channel coding, space-time coding, joint source and channel coding, iterative detection, OFDM, MIMO, cooperative communications, distributed coding, quantum error correction codes and joint wireless-and-optical-fiber communications. He has published over 150 papers and co-authored two John Wiley/IEEE Press books in this field. He is a senior member of the IEEE, a Chartered Engineer and a fellow of the Higher Education Academy in the UK.

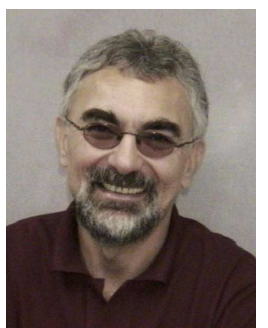

Lajos Hanzo FREng, FIEEE, FIET, Fellow of EURASIP, DSc received his degree in electronics in 1976 and his doctorate in 1983. In 2009 he was awarded the honorary doctorate "Doctor Honoris Causa" by the Technical University of Budapest. During his 35-year career in telecommunications he has held various research and academic posts in Hungary, Germany and the UK. Since 1986 he has been with the School of Electronics and Computer Science, University of Southampton, UK, where he holds the chair in telecommunications. He has successfully supervised $80 \mathrm{PhD}$ students, co-authored $20 \mathrm{John}$ Wiley/IEEE Press books on mobile radio communications totalling in excess of 10000 pages, published 1300 research entries at IEEE Xplore, acted both as TPC and General Chair of IEEE conferences, presented keynote lectures and has been awarded a number of distinctions. Currently he is directing a 100-strong academic research team, working on a range of research projects in the field of wireless multimedia communications sponsored by industry, the Engineering and Physical Sciences Research Council (EPSRC) UK, the European IST Programme and the Mobile Virtual Centre of Excellence (VCE), UK. He is an enthusiastic supporter of industrial and academic liaison and he offers a range of industrial courses. He is also a Governor of the IEEE VTS. During 2008 - 2012 he was the Editor-in-Chief of the IEEE Press and a Chaired Professor also at Tsinghua University, Beijing. His research is funded by the European Research Council's Senior Research Fellow Grant. For further information on research in progress and associated publications please refer to http://www-mobile.ecs.soton.ac.uk. 